\title{
An Integral Estimate for the Gradient for a Class of \\ Nonlinear Elliptic Equations in the Plane
}

\author{
L. Softova
}

\begin{abstract}
An a priori estimate is established for the gradient of the solution to Dirichlet's problem for a class of nonlinear differential equations on a convex domain in the plane. The nonlinear operator is assumed to be elliptic in the sense of Campanato. By virtue of the Leray-Schauder fixed point theorem an existence result for the problem under consideration is derived.

Keywords: Nonlinear elliptic equations, a priori estimates, Aleksandrov-Pucci maximum principle
\end{abstract}

AMS subject classification: $35 \mathrm{R} \mathrm{05,35} \mathrm{J} \mathrm{60,} 35$ B 45

\section{Introduction}

The present paper deals with strong solutions of the Dirichlet problem for second order nonlinear equations of the form

$$
a\left(x, u(x), D u(x), D^{2} u(x)\right)=f(x, u(x), D u(x)) \quad \text { a.e. in } \Omega .
$$

Here $\Omega$ is a bounded, convex and sufficiently smooth domain, and the functions $a$ and $f$ satisfy the Carathéodory condition. Equation ( 0$)$ is assumed to be elliptic in the sense of Campanato (condition (A) below).

Strong solvability results for equation (0) were proved by Bers and Nirenberg [2] under the assumption that $a$ and $f$ are differentiable functions with respect to all their variables. A similar result belongs to Ladyzenskaya and Uralt'zeva when $a$ and $f$ are continuous functions. Imposing an ellipticity condition of special kind on $a$, Campanato was able to handle with operators defined by Carathéodory functions. Local existence results were derived in $[3,4]$ for domains with small Lebesgue measure. Recently, global strong solvability for equation (0) was proved by Palagachev in [8] if the right-hand side $f$ grows strictly subquadratically with respect to the gradient.

Our main goal here is to improve the results in [8] allowing quadratic gradient growth in $f$. Existence of strong solution to the Dirichlet problem for equation $(0)$ is reached by Leray-Schauder's fixed point theorem and is based on a Campanato's theory of

1. Softova: Higher Mil. Transp. School, Dept. Math., 158 Gco Milev Str. 1574 Sofia, Bulgaria

ISSN 0232-2064 / \$2.50 (C) Heldermann Verlatg Berlin 
nearness between operators (see $[3,4]$ ) and on an a priori estimate for the $L^{4}(\Omega)$ norm of the gradient $D u$. In deriving this estimate we use essentially Campanato's ellipticity condition which enables to linearize the equation in a suitable manner and then apply a topological approach due to Amann and Crandall [1].

\section{Setting of the problem and main results}

Let $\Omega \subset \mathbb{R}^{2}$ be a bounded and convex domain of class $C^{2}$. Suppose that $a=a(x, z, p, \xi)$ and $f=f(x, z, p)$ are real-valued functions which satisfy the Carathèodory condition, i.e. they are measurable in $x$ for all $(z, p, \xi) \in \mathbb{R} \times \mathbb{R}^{2} \times \mathbb{R}^{4}$ and continuous in the other variables for almost all $x \in \Omega$. Our aim is to study the following Dirichlet problem for second order nonlinear differential equations

$$
\left.\begin{array}{rlrl}
a\left(x, u, D u, D^{2} u\right) & =f(x, u, D u) & & \text { a.e. in } \Omega \\
u & =0 & & \text { on } \partial \Omega .
\end{array}\right\}
$$

Here the symbols $D u$ and $D^{2} u$ denote the gradient and Hessian matrix of $u$, respectively, and $\mathbb{R}^{4}$ stands for the 4-dimensional space of real and symmetric $(2 \times 2)$-matrices $\xi=\left\{\xi_{i j}\right\}_{i, j=1}^{2}$ with the norm $\|\xi\|=\left(\sum_{i, j=1}^{2} \xi_{i j}^{2}\right)^{\frac{1}{2}}$. We will consider strong solutions of problem (1), i.e. twice weakly differentiable functions $u \in W^{2, q}(\Omega)$ satisfying the equation in (1) a.e. in $\Omega$ and achieving their boundary values in the sense of $W^{1, q}(\Omega)$, i.e. $u \in W_{0}^{1, q}(\Omega)$, for suitable $q \geq 1$.

Concerning the function $a=a(x, z, p, \xi)$ our investigations will be carried out, assuming the validity of the following cllipticity condition introduced by Campanato (see [3]):

(A) There exist positive constants $\alpha, \gamma$ and $\delta, \gamma+\delta<1$, such that

$$
|\operatorname{Tr}(\xi)-\alpha[a(x, z, p, \xi+\tau)-a(x, z, p, \tau)]| \leq \gamma\|\xi\|+\delta|\operatorname{Tr}(\xi)|
$$

for almost all $x \in \Omega$, for all $z \in \mathbb{R}, p \in \mathbb{R}^{2}$ and $\xi, \tau \in \mathbb{R}^{4}$, and $a(x, z, p, 0)=0$.

Concerning the function $f=f(x, z, p)$ we impose the following requirements:

(B) $|f(x, z, p)| \leq f_{1}(|z|)\left(f_{2}(x)+|p|^{2}\right)$, where $f_{1} \in C^{0}\left(\mathbb{R}^{+}\right)$is a positive, monotone non-decreasing function and $f_{2} \in L^{2}(\Omega)$ is positive.

(C) $-\operatorname{sign} z \cdot f(x, z, p) \leq 2 \frac{\sqrt{\operatorname{det}\left|a_{i j}\right|} \sqrt{g(x)}}{h(p)}$ for a.a. $x \in \Omega,|z| \geq M$ and $p \in \mathbb{R}^{2}$, where $a_{i j}(x, z, p, \xi)=\frac{\partial a}{\partial \xi_{i j}}(x, z, p, \xi), a_{i j} \in L^{\infty}\left(\Omega \times \mathbb{R} \times \mathbb{R}^{2} \times \mathbb{R}^{4}\right), g \in L^{1}(\Omega)$ and $h \in L_{\text {loc }}^{\mathbf{l}}\left(\mathbb{R}^{2}\right)$ are positive functions such that $\int_{\Omega} g(x) d x<\int_{\mathbb{R}^{2}} h(p) d p$ (see [10]).

Let us note that, according to [8: Lemma], Campanato's condition (A) ensures that $a=$ $a(x, z, p, \xi)$ is a Lipschitz-continuous function with respect to $\xi$. Hence, in view of the classical Rademacher thcorem, the derivatives $\frac{\partial a}{\partial \xi_{i j}}(x, z, p, \xi)$ exist almost everywhere, and they are essentially bounded.

Now we can formulate our main result. 
Theorem 1 (Gradient estimate). Assume $\Omega \subset \mathbb{R}^{2}$ to be a bounded and convex domain of class $C^{2}$, and let conditions (A) and (B) be fulfilled. Then there exists a constant $C=C\left(\alpha, \gamma, \delta, \partial \Omega, f_{1}, f_{2},\|u\|_{L^{\infty}(\Omega)}\right)$ such that

$$
\|D u\|_{L^{4}(\Omega)} \leq C
$$

for each strong solution $u \in W^{2,2}(\Omega) \cap W_{0}^{1,2}(\Omega)$ of the Dirichlet problem (1).

The a priori gradient estimate already stated allows us to apply the Leray-Schauder fixed point theorem in order to derive strong solvability of problem (1).

Theorem 2 (Existence). Let $\Omega \subset \mathbb{R}^{2}$ be a bounded and convex domain of class $C^{2}$, and let conditions (A), (B) and (C) be satisfied. Then the Dirichlet problem (1) admits a solution $u \in W^{2,2}(\Omega) \cap W_{0}^{1,2}(\Omega)$.

To conclude this section let us note that the strong solution $u$ of problem (1) is a Hölder-continuous function $u \in C^{0, \lambda}(\bar{\Omega})$ for all $\lambda<1$ in view of Sobolev's imbedding theorem. Hence, $u$ attains its boundary values on $\partial \Omega$ continuously.

In addition to the assumptions in Theorem 2, suppose that $a=a(x, z, p, \xi)$ is independent of $z$ and $p, f(x, z, p)$ is non-decreasing in $z$ and Lipschitz continuous with respect to $p$. Then the solution of the Dirichlet problem (1) is unique in the wider class $C^{0}(\bar{\Omega}) \cap W_{\text {loc }}^{2,2}(\Omega)$. We refer to [8: Theorem 2] for the details.

\section{Proofs of the results}

We start with proving the gradient a priori estimate (2). For this goal an approach due to Amann and Crandall [1] will be used.

Let $u \in W^{2,2}(\Omega) \cap W_{0}^{1,2}(\Omega)$ solve the Dirichlet problem (1). The equation in (1) can be rewritten in the form

$$
a\left(x, u, D u, D^{2} u\right)-\frac{f(x, u, D u)\left(f_{2}(x)+|D u|^{2}\right)}{f_{2}(x)+|D u|^{2}}=0
$$

which gives

$$
a\left(x, u, D u, D^{2} u\right)-\frac{f(x, u, D u)|D u|^{2}}{f_{2}(x)+|D u|^{2}}-f_{2}(x) u(x)=\frac{f(x, u, D u) f_{2}(x)}{f_{2}(x)+|D u|^{2}}-f_{2}(x) u(x) .
$$

Now, defining the functions

$$
b(x)=-\frac{f(x, u, D u)}{f_{2}(x)+|D u|^{2}} \quad \text { and } \quad F(x)=\frac{f(x, u, D u) f_{2}(x)}{f_{2}(x)+|D u|^{2}}-f_{2}(x) u(x),
$$

the equation in (1) takes on the form

$$
a\left(x, u, D u, D^{2} u\right)+b(x)|D u|^{2}-f_{2}(x) u(x)=F(x)
$$


where, according to condition (B), we have $|b(x)| \leq f_{1}\left(\|u\|_{L^{\infty}}\right)<\infty$ for a.a. $x \in \Omega$, i.e. $b \in L^{\infty}(\Omega)$ and $F \in L^{2}(\Omega)$. Thus, the Dirichlet problem (1) is equivalent to the following one:

$$
\left.\begin{array}{rlrl}
a\left(x, u, D u, D^{2} u\right)+b(x)|D u|^{2}-f_{2}(x) u(x) & =F(x) & & \text { a.e. in } \Omega \\
u & =0 & & \text { on } \partial \Omega .
\end{array}\right\}
$$

Let $\rho \in[0,1]$ be a parameter and consider the problem

$$
\left.\begin{array}{rlrl}
a\left(x, u, D u, D^{2} v\right)+b(x)|D v|^{2}-f_{2}(x) v(x) & =\rho F(x) & & \text { a.e. in } \Omega \\
v & =0 & & \text { on } \partial \Omega .
\end{array}\right\}
$$

Note that the function $v=0$ solves problem (4) if $\rho=0$. On the other hand, problem (4) coincides with the original problem (3) for $\rho=1$. Thus, if we know in addition uniqueness result for problem (4), then the solution $v$ of problem (4) with $\rho=1$ will coincide with the solution $u$ of problem (3).

Proposition 3. Let $v_{1}, v_{2} \in W^{2,2}(\Omega) \cap W_{0}^{1,2}(\Omega)$ be two solutions of problem (4) corresponding to the respective values $\rho_{1} \leq \rho_{2}$ of the parameter $\rho$. Then

$$
\left\|v_{1}-v_{2}\right\|_{L^{\infty}(\Omega)} \leq\left(\rho_{2}-\rho_{1}\right)\left[f_{1}\left(\|u\|_{L^{\infty}(\Omega)}\right)+\|u\|_{L^{\infty}(\Omega)}\right]
$$

Proof. Clearly, we have

$$
\left.\begin{array}{rlrl}
a\left(x, u, D u, D^{2} v_{1}\right)-a\left(x, u, D u, D^{2} v_{2}\right) & & \\
+b(x)\left[\left|D v_{1}\right|^{2}-\left|D v_{2}\right|^{2}\right]-f_{2}(x)\left[v_{1}(x)-v_{2}(x)\right] & =F(x)\left(\rho_{1}-\rho_{2}\right) & & \text { a.e. in } \Omega \\
v_{1}-v_{2}=0 & & \text { on } \partial \Omega .
\end{array}\right\}
$$

According to [8: Lemma], the function $\xi \rightarrow a(x, z, p, \xi)$ is differentiable a.e. with respect to $\xi$ and the derivatives $\frac{\partial a}{\partial \xi_{i j}}(x, z, p, \xi)(i, j=1,2)$ belong to $L^{\infty}\left(\Omega \times \mathbb{R} \times \mathbb{R}^{2} \times \mathbb{R}^{4}\right)$. Thercfore, we derive from (6)

$$
\begin{aligned}
\int_{0}^{1} & \frac{\partial a}{\partial \xi_{i j}}\left(x, u, D u, s\left(D^{2} v_{1}-D^{2} v_{2}\right)+D^{2} v_{2}\right) D_{i j}\left(v_{1}-v_{2}\right) d s \\
+b(x) & \int_{0}^{1} \frac{\partial}{\partial s}\left|s\left(D v_{1}-D v_{2}\right)+D v_{2}\right|^{2} d s-f_{2}(x)\left[v_{1}(x)-v_{2}(x)\right]=F(x)\left(\rho_{1}-\rho_{2}\right) .
\end{aligned}
$$

Setting $w=v_{1}-v_{2}$ and introducing the notations

$$
\begin{aligned}
A_{i j}(x) & =\int_{0}^{1} \frac{\partial a}{\partial \xi_{i j}}\left(x, u ; D u, s\left(D^{2} v_{1}-D^{2} v_{2}\right)+D^{2} v_{2}\right) d s \\
b_{i}(x) & =2 b(x) \int_{0}^{1}\left[s\left(D_{i} v_{1}-D_{i} v_{2}\right)+D_{i} v_{2}\right] d s,
\end{aligned}
$$


the equation in (6) takes on the form

$$
L w \equiv A_{i j}(x) D_{i j} w+b_{i}(x) D_{i} w-f_{2}(x) w(x)=F(x)\left(\rho_{1}-\rho_{2}\right) .
$$

To apply the Aleksandrov-Pucci maximum principle (see [5: Theorem 9.1]) we need an estimate for the right-hand side $F(x)\left(\rho_{1}-\rho_{2}\right)$ from above:

$$
\begin{aligned}
F(\dot{x}) & =\frac{f(x, u, D u) f_{2}(x)}{f_{2}(x)+|D u|^{2}}-f_{2}(x) u(x) \\
& \leq \frac{|f(x, u, D u)| f_{2}(x)}{f_{2}(x)+|D u|^{2}}+f_{2}(x)|u(x)| \\
& \leq \frac{f_{1}(|u|)\left[f_{2}(x)+|D u|^{2}\right] f_{2}(x)}{f_{2}(x)+|D u|^{2}}+f_{2}(x)|u(x)| \\
& \leq f_{2}(x)\left[f_{1}(|u|)+|u|\right] \\
& \leq f_{2}(x)\left[f_{1}\left(\|u\|_{L^{\infty}(\Omega)}\right)+\|u\|_{L^{\infty}(\Omega)}\right] .
\end{aligned}
$$

Since $\left(\rho_{1}-\rho_{2}\right)$ is negative, we get

$$
\begin{aligned}
L w & \geq\left(\rho_{1}-\rho_{2}\right) f_{2}(x)\left[f_{1}\left(\|u\|_{L^{\infty}(\Omega)}\right)+\|u\|_{L^{\infty}(\Omega)}\right] \\
& =-f_{2}(x)\left(\rho_{2}-\rho_{1}\right)\left[f_{1}\left(\|u\|_{L^{\infty}(\Omega)}\right)+\|u\|_{L^{\infty}(\Omega)}\right] .
\end{aligned}
$$

Denoting $M=\left(\rho_{2}-\rho_{1}\right)\left[f_{1}\left(\|u\|_{L^{\infty}(\Omega)}\right)+\|u\|_{L^{\infty}(\Omega)}\right]$, it is clear that

$$
L M=-f_{2}(x)\left(\rho_{2}-\rho_{1}\right)\left[f_{1}\left(\|u\|_{L^{\infty}(\Omega)}\right)+\|u\|_{L^{\infty}(\Omega)}\right]
$$

and $L w \geq L M$. Now applying the Aleksandrov-Pucci maximum principle to the problem

$$
\left.\begin{array}{rl}
L(w-M) \geq 0 & \text { a.e. in } \Omega \\
w-M \leq 0 & \text { on } \partial \Omega
\end{array}\right\}
$$

we get $w-M \leq 0$ a.e. in $\Omega$ and hence $w \leq M$. Considering the same problem with $-w$ instead of $w$, we get an estimate for $w$ from below, that yields $w \geq-M$, whence $\|w\|_{L^{\infty}(\Omega)} \leq M$

Corollary 4. If problem (4) has a solution $v \in W^{2,2}(\Omega) \cap W_{0}^{1,2}(\Omega)$ for some $\rho \in$ $[0,1]$, then it is a unique solution.

Proof. It follows immediately from (5) putting $\rho_{1}=\rho_{2}$

We are in a position now to prove estimate (2). Let $\rho_{1}<\rho_{2}$ and denote the corresponding solutions of problem (4) by $v_{1}, v_{2} \in W^{2,2}(\Omega) \cap W_{0}^{1,2}(\Omega)$. As above we set $w=v_{1}-v_{2}$ and consider the problem

$$
\begin{aligned}
a\left(x, u, D u, D^{2} v_{1}\right) & -a\left(x, u, D u, D^{2} v_{2}\right) \\
& =F(x)\left(\rho_{1}-\rho_{2}\right) \\
& -b(x)\left[\left|D v_{1}\right|^{2}-\left|D v_{2}\right|^{2}\right]+f_{2}(x)\left[v_{1}(x)-v_{2}(x)\right] \quad \text { a.e. in } \Omega \\
v_{1}-v_{2} & =0 \text { on } \partial \Omega
\end{aligned}
$$


Introduce the new function

$$
G(x)=\left(\rho_{1}-\rho_{2}\right) F(x)-b(x)\left[\left|D v_{1}\right|^{2}-\left|D v_{2}\right|^{2}\right]+f_{2}(x) w(x)
$$

and consider the equation

$$
\Delta w=\Delta w-\alpha\left[a\left(x, u, D u, D^{2} v_{1}\right)-a\left(x, u, D u, D^{2} v_{2}\right)\right]+\alpha G(x)
$$

Having in mind that $\operatorname{Tr}\left(D^{2} w\right)=\Delta w$, condition (A), and Young's inequality we get

$$
\begin{aligned}
|\Delta w|^{2}= & \left|\Delta w-\alpha\left[a\left(x, u, D u, D^{2} v_{1}\right)-a\left(x, u, D u, D^{2} v_{2}\right)\right]\right|^{2}+\alpha^{2}|G(x)|^{2} \\
& +2\left|\Delta w-\alpha\left[a\left(x, u, D u, D^{2} v_{1}\right)-a\left(x, u, D u, D^{2} v_{2}\right)\right]\right||G(x)| \\
\leq & \left|\Delta w-\alpha\left[a\left(x, u, D u, D^{2} v_{1}\right)-a\left(x, u, D u, D^{2} v_{2}\right)\right]\right|^{2}+\alpha^{2}|G(x)|^{2} \\
& +\varepsilon\left|\Delta w-\alpha\left[a\left(x, u, D u, D^{2} v_{1}\right)-a\left(x, u, D u, D^{2} v_{2}\right)\right]\right|^{2}+\frac{1}{\varepsilon}|G(x)|^{2} \\
\leq & (1+\varepsilon)\left(\gamma\left\|D^{2} w\right\|+\delta|\Delta w|\right)^{2}+C(\varepsilon, \alpha)|G(x)|^{2} \\
\leq & (1+\varepsilon) \gamma(\gamma+\delta)\left\|D^{2} w\right\|^{2}+(1+\varepsilon) \delta(\delta+\gamma)|\Delta w|^{2}+C(\varepsilon, \alpha)|G(x)|^{2}
\end{aligned}
$$

for arbitrary $\varepsilon>0$. Thus

$$
\begin{aligned}
\int_{\Omega}|\Delta w|^{2} d x \leq & \int_{\Omega}(1+\varepsilon) \gamma(\gamma+\delta)\left\|D^{2} w\right\|^{2} d x \\
& +\int_{\Omega}(1+\varepsilon) \delta(\delta+\gamma)|\Delta w|^{2} d x+C(\alpha, \varepsilon) \int_{\Omega}|G(x)|^{2} d x
\end{aligned}
$$

Since $w \in W^{2,2}(\Omega) \cap W_{0}^{1,2}(\Omega)$, and $\Omega$ is a convex domain, the Miranda-Talenti inequality (see $[6,9])$

$$
\int_{\Omega}\left\|D^{2} w\right\|^{2} d x \leq \int_{\Omega}|\Delta w|^{2} d \dot{x}
$$

can be applied. It follows

$$
\int_{\Omega}|\Delta w|^{2} d x \leq(1+\varepsilon)(\gamma+\delta)^{2} \int_{\Omega}|\Delta w|^{2} d x+C(\alpha, \varepsilon) \int_{\Omega}|G(x)|^{2} d x
$$

and if $\varepsilon>0$ is so small that $(1+\varepsilon)(\gamma+\delta)^{2}<1$, we obtain

$$
\left[1-(1+\varepsilon)(\gamma+\delta)^{2}\right] \int_{\Omega}|\Delta w|^{2} d x \leq C(\alpha, \varepsilon) \int_{\Omega}|G(x)|^{2} d x
$$

Therefore, for a constant $C_{1}$, depending on $\alpha, \varepsilon, \gamma$ and $\delta$, it results

$$
\int_{\Omega}|\Delta w|^{2} d x \leq C_{1}(\alpha, \varepsilon, \gamma, \delta) \int_{\Omega}|G(x)|^{2} d x
$$


Using (8) once again, we get an estimate for $D^{2} w$ :

$$
\int_{\Omega}\left\|D^{2} w\right\|^{2} d x \leq C_{1}(\alpha, \varepsilon, \gamma, \delta) \int_{\Omega}|G(x)|^{2} d x
$$

i.e.

$$
\left\|D^{2} w\right\|_{L^{2}(\Omega)}^{2} \leq C_{1}(\alpha, \varepsilon, \gamma, \delta)\|G(x)\|_{L^{2}(\Omega)}^{2} .
$$

Thus, the function $w$ satisfies the inequality

$$
\begin{aligned}
& \|w\|_{W^{2,2}(\Omega)} \\
& \quad \leq C\left(\|F(x)\|_{L^{2}(\Omega)}+\left\|b(x)\left[\left|D v_{2}\right|^{2}-\left|D v_{1}\right|^{2}\right]\right\|_{L^{2}(\Omega)}+\left\|f_{2} w\right\|_{L^{2}(\Omega)}\right) \\
& \quad \leq C_{2}\left(\left\|f_{2}\right\|_{L^{2}(\Omega)},\|w\|_{L^{\infty}(\Omega)},\|b(x)\|_{L^{\infty}(\Omega)}\right)\left(1+\left\|D v_{1}\right\|_{L^{4}(\Omega)}^{2}+\|D w\|_{L^{4}(\Omega)}^{2}\right) .
\end{aligned}
$$

The $L^{4}$-norm of $D w$ in the right-hand side above can be estimated by the help of Gagliardo-Nirenberg's inequality [7]

$$
\|D w\|_{L^{4}(\Omega)} \leq K^{\frac{1}{2}}\left\|D^{2} w\right\|_{L^{2}(\Omega)}^{\frac{1}{2}}\|w\|_{L^{\infty}(\Omega)}^{\frac{1}{2}} .
$$

In other words, utilizing (5) we derive

$$
\begin{aligned}
\|D w\|_{L^{4}(\Omega)}^{2} & \leq K\left\|D^{2} w\right\|_{L^{2}(\Omega)}\|w\|_{L^{\infty}(\Omega)} \\
& \leq K\left(\rho_{2}-\rho_{1}\right)\left[f_{1}\left(\|u\|_{L^{\infty}(\Omega)}\right)+\|u\|_{L^{\infty}(\Omega)}\right]\left\|D^{2} w\right\|_{L^{2}(\Omega)}
\end{aligned}
$$

which leads to

$$
\begin{aligned}
\|w\|_{W^{2,2}(\Omega)} \leq & C_{2}\left\{1+\left\|D v_{1}\right\|_{L^{4}(\Omega)}^{2}\right. \\
& \left.+K\left(\rho_{2}-\rho_{1}\right)\left[f_{1}\left(\|u\|_{L^{\infty}(\Omega)}\right)+\|u\|_{L^{\infty}(\Omega)}\right]\left\|D^{2} w\right\|_{L^{2}(\Omega)}\right\} .
\end{aligned}
$$

Thus, having in mind $\left\|D^{2} w\right\|_{L^{2}(\Omega)} \leq\|w\|_{W^{2,2}(\Omega)}$, we get

$$
\left\|D^{2} w\right\|_{L^{2}(\Omega)} \leq C_{3}\left(1+\left\|D v_{1}\right\|_{L^{4}(\Omega)}^{2}\right)
$$

assuming in addition $\rho_{2}-\rho_{1} \leq \tau$ to be so small that $C_{2} K\left(\rho_{2}-\rho_{1}\right)\left[f_{1}\left(\|u\|_{L^{\infty}(\Omega)}\right)+\right.$ $\left.\|u\|_{L^{\infty}(\Omega)}\right]<1$. Hence

$$
\begin{aligned}
\left\|D v_{2}\right\|_{L^{4}(\Omega)}^{2} \leq & \left\|D v_{1}\right\|_{L^{4}(\Omega)}^{2} \\
& +K\left(\rho_{2}-\rho_{1}\right)\left[f_{1}\left(\|u\|_{L^{\infty}(\Omega)}\right)+\|u\|_{L^{\infty}(\Omega)}\right] C_{3}\left(1+\left\|D v_{1}\right\|_{L^{4}(\Omega)}^{2}\right) \\
\leq & \left\|D v_{1}\right\|_{L^{4}(\Omega)}^{2}+\|D w\|_{L^{4}(\Omega)}^{2} \\
\leq & C_{4}+C_{5}\left\|D v_{1}\right\|_{L^{4}(\Omega)}^{2} .
\end{aligned}
$$

Bound (9) means that, if for some $\rho_{1} \in[0,1]$ we have an a priori estimate for the respective solution $v_{1}$ of problem (4), we can get an estimate for the other solution $v_{2}$ of the same problem with $\rho_{2}>\rho_{1}\left(\rho_{2} \in[0,1]\right)$ if $\rho_{2}-\rho_{1} \leq \tau$. 
To proceed further, we set $\rho_{1}=0$ and $\rho_{2}=\tau$. In view of the uniqueness result (Corollary 4), the solution $v_{0}$ of problem (4) with $\rho=0$ is equal to zero and thus (9) yields

$$
\left\|D v_{\tau}\right\|_{L^{4}(\Omega)}^{2} \leq C_{4}
$$

whenever there exists the solution $v_{\tau}$ of problem (4) with $\rho=\tau$. Thus, choosing $\rho_{1}=k \tau$ and $\rho_{2}=(k+1) \tau$, with $k=0,1, \ldots, m-1$, and repeating the above procedure $m$ times, we derive the desired estimate $(2):\|D u\|_{L^{4}(\Omega)} \leq C_{6}$.

It remains to prove strong solvability of problem (4) with $\rho=\tau$. This will be carried out by using the Leray-Schauder fixed point theorem. Consider problem (4) with $\rho=\tau$, i.e.

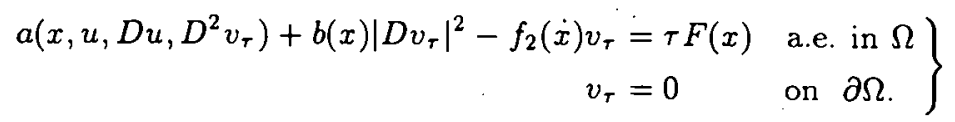

We define the operator

$$
\mathcal{M}:[0,1] \times W^{1,4}(\Omega) \longrightarrow W^{2,2}(\Omega) \cap W_{0}^{1,2}(\Omega)
$$

as follows. For all $\sigma \in[0,1]$ and $y \in W^{1,4}(\Omega)$ consider the problem

$$
\left.\begin{array}{rlrl}
a\left(x, u, D u, D^{2} z\right) & =\sigma\left[\tau F(x)-b(x)|D y|^{2}+f_{2}(x) y\right] & & \text { a.e. in } \Omega \\
z & =0 & & \text { on } \partial \Omega .
\end{array}\right\}
$$

In order to ensure solvability of this problem we need to show that the right-hand side of the equation above belongs to $L^{2}(\Omega)$. In fact, $F \in L^{2}(\Omega)$ and $b \in L^{\infty}(\Omega)$ as it was mentioned. Further on, $y \in W^{1,4}(\Omega)$ and thus $|D y|^{2} \in L^{2}(\Omega)$. Finally, $y \in W^{1,4}(\Omega) \subset C^{0}(\bar{\Omega})$ by virtue of Sobolev's imbedding theorem and therefore

$$
\int_{\Omega} f_{2}^{2}(x) y^{2}(x) d x \leq\left(\sup _{\Omega}|y(x)|\right)^{2} \int_{\Omega}\left|f_{2}(x)\right|^{2} d x<\infty .
$$

According to [4: Theorem 3] or [3: Theorem 4.4] and Campanato's condition $(A)$ of ellipticity, problem (12) has a unique solution $z \in W^{2,2}(\Omega) \cap W_{0}^{\mathbf{i}, 2}(\Omega)$. This way we defined an operator

$$
\mathcal{M}:[0,1] \times W^{1,4}(\Omega) \longrightarrow W^{2,2}(\Omega) \cap W_{0}^{1,2}(\Omega)
$$

by the formula $\mathcal{M}(\sigma, y)=z$. It is casily seen that each fixed point of the operator $\mathcal{M}(1, \cdot)$ is a solution of problem (12). The existence of such fixed point will follow from Leray-Schauder's theorem. The condition $a(x, z, p, 0)=0$ as required above shows that $\mathcal{M}(0, y)=0$ for each $y \in W^{1,4}(\Omega)$. The operator $\mathcal{M}$ is a continuous one as it is proved in [8]. Moreover, $\mathrm{M}$ is a compact operator considering it as a mapping from $[0,1] \times W^{1,4}(\Omega)$ into $W^{1,4}(\Omega)$. The last assertion is a consequence of the fact that $W^{2,2}(\Omega)$ is compactly imbedded into $W^{1,4}(\Omega)$ (Rellich's theorem).

Finally, (10) gives an a priori estimate with a constant independent of $v_{\tau}$ and $\sigma$ for each solution $v_{\tau} \in W^{2,2}(\Omega) \cap W_{0}^{1,2}(\Omega) \subset W^{1,4}(\Omega)$ of the equation $\mathcal{M}\left(\sigma, v_{\tau}\right)=v_{\tau}$ which is equivalent to the Dirichlet problem

$$
\left.\begin{array}{rlrl}
a\left(x, u, D u, D^{2} v_{\tau}\right) & =\sigma\left[\tau F(x)-b(x)\left|D v_{\tau}\right|^{2}+f_{2}(x) v_{\tau}\right] & & \text { a.e. in } \Omega \\
v_{\tau} & =0 & & \text { on } \partial \Omega .
\end{array}\right\}
$$

Hence Leray-Schauder's theorem implies the existence of a fixed point of $\mathcal{M}(1, \cdot)$ which is a solution of problem (4) with $\rho \doteq \tau$. This completes the proof of Theorem 1 
The proof of the existence result (Theorem 2) is similar to the proof of [8: Theorem 1) and it makes use of Leray-Schauder's fixed point principle. However, in addition to the gradient estimate (2) we need an a priori bound for $\|u\|_{L^{\infty}}(\Omega)$.

Proposition 5. Let conditions (A) and (C) hold. Then each solution $u \in W^{2,2}(\Omega) \cap$ $W_{0}^{1,2}(\Omega)$ of problem (1) satisfies the estimate

$$
\|u\|_{L^{\infty}(\Omega)} \leq M+R \operatorname{diam} \Omega
$$

where $R$ is such that $\int_{B_{R}} h(p) d p=\int_{\Omega} g(x) d x$ and $B_{R}$ is a ball with center at the origin and radius $R$.

Proof. Since $a(x, z, p, 0)=0$, the function $u \in W_{0}^{1,2}(\Omega) \cap W^{2,2}(\Omega)$ solves the problem

$$
\left.\begin{array}{rlrl}
a^{i j}(x) D_{i j} u & =f(x, u, D u) & & \text { a.e. in } \Omega \\
u & =0 & & \text { on } \partial \Omega
\end{array}\right\}
$$

where $a^{i j} \in L^{\infty}(\Omega)$

$$
a^{i j}(x)=\int_{0}^{1} \frac{\partial a}{\partial \xi_{i j}}\left(x, u(x), D u(x), s D^{2} u(x)\right) d s
$$

(see [8: Lemma]). Hence, the statement of Proposition 5 follows from condition (C) and [10: Theorem 2.6.1]

Acknowledgements. The results presented here were obtained during the author's visit at the Department of Mathematics, University of Catania. The author wishes to express his deep gratitude to all staff of the Department for the hospitality, and especially to Prof. A. Maugeri for his kindness and useful discussions.

\section{References}

[1] Amann, H. and M. Crandall: On some existence theorems for semi-linear elliptic equations. Indiana Univ. Math. J. 27 (1978), $779-790$.

[2] Bers, L. and L. Nirenberg: On linear and non-linear elliptic boundary value problems in the plane. In: Atti del Convegno Intern. sulle Equazioni Lineari alle Derivate Parziali. Trieste: Edizioni Cremonese 1955, pp. 141 - 167.

[3] Campanato, S.: Sistemi differenziali del $2^{\circ}$ ordine di tipo ellitico. Quaderno No 1 del Dottorato di Ricerca in Matematica, Univ. degli Studi di Catania, 1991.

[4] Campanato, S.: Non variational differential systems. A condition for local existence and uniqueness. Ricerche di Matematica (Supplemento, Int. Symp. "R. Caccioppoli") 70 (1991), 129 -- 140.

[5] Gilbarg, D. and N. S. Trudinger: Elliptic Partial Differential Equations of Second Order. 2nd edition. Berlin: Springer-Verlag 1983.

[6] Miranda, C.: Su di una particulare equazione ellitica del $2^{\circ}$ ordine a coefficienti discontinui. Anal. Sti. Univ. "Al. I. Cura", Jasi Sert. Ib. Fiz. 11 (1965), $209-215$. 
[7] Nirenberg, L.: On elliptic partial differential equations. Ann. Scuola Norm. Sup. Pisa (3) 13 (1959), 115 - 162.

[8] Palagachev, D.: Global strong solvability of Dirichlet problem for a class of nonlinear elliptic equations in the plane. Le Matematiche 48 (1993), $311-321$.

[9] Talenti, G.: Equazioni lineari ellittiche in due variabili. Le Matematiche 21 (1966), 339 376.

[10] Trudinger, N. S.: Lectures on Non-Linear Elliptic Equations. Lect. Notes Math. Inst. Nankai Univ., Tianjin (China) 1986.

Received 13.11.1996 